\title{
Clinical Importance of Obesity Versus the Metabolic Syndrome in Cardiovascular Risk in Women A Report From the Women's Ischemia Syndrome Evaluation (WISE) Study
}

\author{
Kevin E. Kip, PhD; Oscar C. Marroquin, MD; David E. Kelley, MD; B. Delia Johnson, PhD; \\ Sheryl F. Kelsey, PhD; Leslee J. Shaw, PhD; William J. Rogers, MD; Steven E. Reis, MD
}

Background - Obesity and the metabolic syndrome frequently coexist. Both are associated with cardiovascular disease (CVD). However, the contribution of obesity to cardiovascular risk, independent of the presence of the metabolic syndrome, remains controversial.

Methods and Results-From the WISE study, 780 women referred for coronary angiography to evaluate suspected myocardial ischemia were classified by body mass index (BMI; $<24.9=$ normal, $\mathrm{n}=184 ; \geq 25.0$ to $\leq 29.9=$ overweight, $\mathrm{n}=269 ; \geq 30.0=$ obese, $\mathrm{n}=327)$ and presence $(\mathrm{n}=451)$ or absence $(\mathrm{n}=329)$ of the metabolic syndrome, further classified by diabetes status. Prevalence of significant angiographic coronary artery disease (CAD; $\geq 50 \%$ stenosis) and 3-year risk of CVD were compared by BMI and metabolic status. The metabolic syndrome and BMI were strongly associated, but only metabolic syndrome was associated with significant CAD. Similarly, unit increases in BMI (normal to overweight to obese) were not associated with 3-year risk of death (adjusted hazard ratio [HR] 0.92, 95\% CI 0.59 to 1.51) or major adverse cardiovascular event (MACE: death, nonfatal myocardial infarction, stroke, congestive heart failure; adjusted HR $0.95,95 \%$ CI 0.71 to 1.27 ), whereas metabolic status (normal to metabolic syndrome to diabetes) conferred an approximate 2-fold adjusted risk of death (HR 2.01, 95\% CI 1.26 to 3.20) and MACE (HR 1.88, 95\% CI 1.38 to 2.57). Levels of C-reactive protein (hs-CRP) were more strongly associated with metabolic syndrome than BMI but were not independently associated with 3-year risk of death or MACE.

Conclusions - The metabolic syndrome but not BMI predicts future cardiovascular risk in women. Although it remains prudent to recommend weight loss in overweight and obese women, control of all modifiable risk factors in both normal and overweight persons to prevent transition to the metabolic syndrome should be considered the ultimate goal. (Circulation. 2004;109:706-713.)

Key Words: cardiovascular diseases $\boldsymbol{\square}$ follow-up studies $\boldsymbol{\square}$ inflammation $\boldsymbol{\square}$ obesity $\boldsymbol{\square}$ women

$\mathrm{I}_{\mathrm{o}}^{\mathrm{n}}$ the United States, more than $60 \%$ of adult women are overweight. During the past 3 decades, the prevalence of obesity has doubled from approximately $15 \%$ to $30 \% .^{1,2}$ Obesity is associated with conventional cardiovascular risk factors (eg, hypertension, dyslipidemia, and diabetes mellitus), ${ }^{3,4}$ novel risk factors (eg, inflammatory markers such as high-sensitivity C-reactive protein [hs-CRP] and interleukin-6 [IL-6]), ${ }^{5}$ and coronary artery endothelial dysfunction. ${ }^{6}$ These associations provide a plausible biological link to epidemiological observations that indicate that obesity is associated with increased risks of both nonfatal and fatal cardiovascular events in women. ${ }^{7-10}$ However, there is substantial heterogeneity of metabolic abnormalities in obese patients, and there remains controversy as to the role of obesity as an independent etiologic factor for incident cardiovascular disease. ${ }^{5}$

Abdominal adiposity is 1 of 5 clinical criteria (including hypertriglyceridemia, low HDL cholesterol, hypertension, and fasting hyperglycemia) that when $\geq 3$ are present define the metabolic syndrome as set forth by the National Cholesterol Education Program (NCEP) Adult Treatment Panel (ATP) III. ${ }^{11}$ The prevalence of the metabolic syndrome currently exceeds $20 \%$ of individuals $\geq 20$ years of age and $40 \%$ of the population $>40$ years of age. ${ }^{12}$ Like obesity, the presence of the metabolic syndrome has been associated with risks of developing diabetes ${ }^{13}$ and cardiovascular dis-

Received December 17, 2003; accepted December 17, 2003

From the Department of Epidemiology (K.E.K., B.D.J., S.F.K.), University of Pittsburgh, Pittsburgh, Pa; University of Pittsburgh Medical Center (O.C.M., S.E.R.), Cardiovascular Institute, Pittsburgh, Pa; University of Pittsburgh School of Medicine (D.E.K.), Department of Medicine, Pittsburgh, Pa; Atlanta Cardiovascular Research Institute (L.J.S.), Atlanta, Ga; and University of Alabama at Birmingham (W.J.R.), Department of Medicine, Birmingham, Ala.

Correspondence to Kevin E. Kip, PhD, University of Pittsburgh, Graduate School of Public Health, 130 DeSoto St, 127 Parran Hall, Pittsburgh, PA 15261. E-mail kipk@edc.pitt.edu

(C) 2004 American Heart Association, Inc. 
ease. ${ }^{14-17}$ Insulin resistance is believed to play a central pathophysiological role in the metabolic syndrome. ${ }^{18}$ However, obesity and the metabolic syndrome/insulin resistance do not uniformly coexist; a significant proportion of persons defined as obese do not develop insulin resistance, and, conversely, insulin resistance can be present in lean individuals. ${ }^{16}$

Chronic subclinical inflammation, which is associated with cardiovascular risk, is believed to be part of the metabolic syndrome. ${ }^{19}$ Epidemiological studies indicate that the predictive power of the metabolic syndrome for cardiovascular disease may be enhanced by the presence of inflammation manifested by high levels of hs-CRP. ${ }^{20}$ Despite these observations, measures of hs-CRP are not currently included in the definition of the metabolic syndrome. ${ }^{21}$ This is particularly relevant to the cardiovascular risk stratification of women, in whom high levels of hs-CRP are associated with development of the metabolic syndrome independent of levels of adiposity. ${ }^{22}$

In the present study of a cohort of women with suspected myocardial ischemia, we evaluated the relative influence of body weight per se versus the metabolic syndrome on the prevalence of significant angiographic coronary artery disease (CAD), as well as incident cardiovascular disease. Furthermore, we explored the potential role of inflammation in the predictive value of body weight and metabolic status.

\section{Methods}

\section{Study Population}

The study population consisted of 780 (83\%) of 944 women enrolled in the Women's Ischemia Syndrome Evaluation (WISE) study, aged 21 to 86 years, in whom the presence or absence of the metabolic syndrome (defined below) could be determined. By study protocol, all women were clinically referred for coronary angiography to evaluate suspected myocardial ischemia at 1 of 4 sites (University of Alabama at Birmingham; University of Florida, Gainesville; University of Pittsburgh, Pittsburgh, Pa; and Allegheny General Hospital, Pittsburgh, Pa). ${ }^{23}$ Exclusion criteria included pregnancy, cardiomyopathy, New York Heart Association class IV angina, congestive heart failure, recent myocardial infarction or revascularization, and any contraindications to provocative testing. All subjects provided informed consent and completed research forms approved by the institutional review board at their local WISE clinical site. On enrollment in the study, each woman had a baseline evaluation that included collection of demographic information, risk factors for $\mathrm{CAD}$, medication use, medical and reproductive history, symptom and psychosocial evaluation, a physical examination with blood pressure and physical measurements, and sampling of blood in the fasting state for lipid, glucose, insulin, reproductive hormone, and inflammatory marker core laboratory evaluations.

\section{Classification of Metabolic Status}

The ATP III criteria ${ }^{11}$ were used to classify study participants as being with or without the metabolic syndrome based on the presence or absence of $\geq 3$ of the following factors: (1) waist circumference $>88 \mathrm{~cm}$, (2) fasting triglycerides $>150 \mathrm{mg} / \mathrm{dL}$ (measured by enzymatic assay at the WISE core lipid laboratory), (3) HDL cholesterol (HDL-C) $<50 \mathrm{mg} / \mathrm{dL}$, (4) hypertension (systolic blood pressure $\geq 130 \mathrm{~mm} \mathrm{Hg}$, diastolic blood pressure $\geq 85 \mathrm{~mm} \mathrm{Hg}$, or use of antihypertensive drug therapy), and (5) fasting glucose $\geq 110$ $\mathrm{mg} / \mathrm{dL}$. Women were classified into 3 mutually exclusive groups consisting of those with a history of treated diabetes (past use of oral hypoglycemic agents or insulin; $n=192 ; 25 \%$ ); those without treated diabetes who met the definition of the metabolic syndrome $(n=259$;
$33 \%)$, and metabolic "normal" subjects $(n=329 ; 42 \%)$. In some analyses, women with a history of diabetes or the metabolic syndrome were combined ("dysmetabolic") and compared with metabolic "normal" women.

\section{Classification of Body Mass Index}

Body mass index (BMI) was calculated as body weight in kilograms divided by the square of the height in meters $\left(\mathrm{kg} / \mathrm{m}^{2}\right)$. Normal weight was defined as $\mathrm{BMI} \leq 24.9(\mathrm{n}=184 ; 24 \%)$, overweight was defined as $\mathrm{BMI} \geq 25.0$ to $\leq 29.9(\mathrm{n}=269 ; 34 \%)$, and obesity was defined as $\mathrm{BMI} \geq 30.0(\mathrm{n}=327 ; 42 \%) .{ }^{24} \mathrm{We}$ did not form a separate analysis group for "underweight" subjects (BMI $<18.5)$, because only 13 subjects ( $1.7 \%$ of study cohort) met this definition, and the results were essentially unchanged when these subjects were excluded. We had the opportunity to use waist circumference as a measure of adiposity in lieu of or an as adjunct to BMI but chose to use BMI because it is not directly part of the definition of the metabolic syndrome.

\section{Measurement of Inflammatory Markers}

Plasma sampled at baseline was frozen at $-70^{\circ} \mathrm{C}$ for subsequent measurement of inflammatory markers. IL-6 levels were measured from plasma collected at study entry with a commercially available ELISA kit (Quantikine hs human IL-6, R\&D Systems). Levels of hs-CRP were measured by a high-sensitivity method on the Hitachi 911 analyzer with reagents from Denka Seiken and previously validated techniques. ${ }^{25}$

\section{Assessment of Angiographic CAD}

Quantitative analysis of coronary angiograms was performed offline at the WISE angiographic core laboratory (Rhode Island Hospital, Providence, RI) by investigators blinded to all other subject data. ${ }^{26}$ Luminal diameter was measured at all stenoses and at nearby reference segments with an electronic cine projector-based crosshair technique (Vanguard Instrument Corporation). The presence of 1 or more stenoses $\geq 50 \%$ in diameter was considered significant CAD, maximum diameter stenosis $20 \%$ to $49 \%$ was considered minimal CAD, and $<20 \%$ stenosis in all coronary arteries was considered no CAD.

\section{Ascertainment of Cardiovascular Events}

Follow-up for the occurrence of untoward cardiovascular events was obtained by annual telephone and/or mail contact. The primary clinical outcomes of interest were death or the composite end point of major adverse cardiovascular events (MACE; death, nonfatal myocardial infarction, stroke, or congestive heart failure). Among the 739 women who did not die during the 3-year follow-up, 87\% had $\geq 1$ year of follow-up, $81 \%$ had $\geq 2$ years of follow-up, and $67 \%$ had $\geq 3$ years of follow-up. The median length of follow-up was 3.5 years (interquartile range 2.8 to 4.7 years) among the 739 surviving women $(94.7 \%$ of the total cohort), and 1.3 years for the 41 nonsurviving women (interquartile range 0.44 to 2.2 years).

\section{Statistical Analysis}

Differences in baseline demographic and clinical characteristics among the 3 BMI groups were assessed by $\chi^{2}$ tests for categorical variables and Student $t$ test or Wilcoxon rank sum tests for continuous variables. The latter nonparametric method was used due to relatively high skew of some baseline clinical variables, including lipids and inflammatory markers. Logistic regression analysis was used to obtain adjusted estimates of the odds of having significant angiographic CAD in relation to levels of BMI and metabolic status. The Kaplan-Meier method was used to estimate 3-year cumulative incidence rates of death and MACE, with the log-rank statistic used to assess differences by levels of BMI and metabolic status. Participants who did not experience the clinical outcome of interest were censored at either 3 years or the last date of follow-up before 3 years. Proportional hazards regression was used to estimate adjusted 3-year relative risks of death and MACE in relation to levels of BMI and metabolic status. Covariates in adjusted models included 
TABLE 1. Baseline Characteristics of Study Cohort by BMI

\begin{tabular}{|c|c|c|c|c|}
\hline \multirow[b]{2}{*}{ Baseline Characteristic } & \multicolumn{4}{|c|}{ BMI } \\
\hline & $\begin{array}{l}\text { Normal }(\leq 24.9) \\
\quad(n=184)\end{array}$ & $\begin{array}{l}\text { Overweight (25 to } \leq 29.9) \\
\qquad(\mathrm{n}=269)\end{array}$ & $\begin{array}{l}\text { Obese }(\geq 30) \\
\quad(n=327)\end{array}$ & $P$ \\
\hline Age, y (mean $\pm S D)$ & $59(13)$ & $58(11)$ & $57(11)$ & 0.12 \\
\hline White race, $\%$ & 88.0 & 82.9 & 73.4 & $<0.0001$ \\
\hline Postmenopausal, \% & 76.8 & 73.2 & 72.8 & 0.60 \\
\hline \multicolumn{5}{|l|}{ Hormone replacement therapy, \% } \\
\hline Ever & 56.6 & 52.1 & 48.8 & 0.24 \\
\hline Current & 40.1 & 35.4 & 30.2 & 0.07 \\
\hline Aspirin use, \% & 58.2 & 62.6 & 59.6 & 0.61 \\
\hline Lipid-lowering statin use, \% & 27.2 & 24.6 & 28.1 & 0.62 \\
\hline Antihypertensive drug use, \% & 39.1 & 44.0 & 58.4 & $<0.0001$ \\
\hline Cigarette smoking, \% & & & & 0.002 \\
\hline Never & 44.6 & 47.8 & 48.6 & \\
\hline Former & 27.7 & 29.9 & 37.6 & \\
\hline Current & 27.7 & 22.4 & 13.8 & \\
\hline Physical activity, mean (SD)* & $2.6(0.7)$ & $2.5(0.6)$ & $2.4(0.7)$ & 0.005 \\
\hline History of CHF, \% & 8.2 & 8.7 & 10.2 & 0.70 \\
\hline Family history of CAD, \% & 60.0 & 66.3 & 68.7 & 0.14 \\
\hline \multicolumn{5}{|c|}{ Blood pressure, mm Hg, mean (SD) } \\
\hline Systolic & $133(23)$ & $135(19)$ & $141(21)$ & $<0.0001$ \\
\hline Diastolic & $74(11)$ & $77(10)$ & $79(11)$ & $<0.0001$ \\
\hline \multicolumn{5}{|l|}{ Cholesterol, mg/dL, mean (SD)† } \\
\hline Total & $197(46)$ & $198(45)$ & $191(45)$ & 0.20 \\
\hline HDL & $59(15)$ & $53(12)$ & $52(12)$ & $<0.0001$ \\
\hline LDL & $110(41)$ & $113(41)$ & $106(41)$ & 0.16 \\
\hline Triglycerides, mean (SD)† & $140(106)$ & $158(122)$ & $167(137)$ & 0.0007 \\
\hline HsCRP, mg/dL, mean (SD)† & $0.58(1.10)$ & $0.97(2.00)$ & $0.89(1.18)$ & $<0.0001$ \\
\hline IL-6, pg/mL, mean (SD)† & $3.9(4.5)$ & $4.5(4.9)$ & $4.7(3.8)$ & $<0.0001$ \\
\hline Metabolic status, \% & & & & $<0.0001$ \\
\hline Normal & 71.7 & 44.6 & 23.5 & \\
\hline Dysmetabolic $\ddagger$ & 15.8 & 34.9 & 41.6 & \\
\hline Diabetic $\ddagger$ & 12.5 & 20.5 & 34.9 & \\
\hline
\end{tabular}

age and race, as well as those with a probability value of $<0.10$, as determined by forward stepwise regression. The proportional hazards assumption of invariant relative risk was tested and found to be satisfactory for all models constructed.

\section{Results}

\section{Baseline Characteristics by BMI}

Compared with normal and overweight women, obese women were significantly less likely to be white and to smoke cigarettes at study entry and significantly more likely to be taking antihypertensive medications and to have higher levels of triglycerides (Table 1). In addition, mean HDL cholesterol levels were lower in overweight $($ mean $=53)$ and obese $($ mean $=52)$ women than in women with normal BMI (mean $=59, P<0.0001$ ), whereas levels of the proinflamma- tory markers hs-CRP and IL-6 were significantly higher $(P<0.0001)$ in overweight and obese women than in women with normal BMI. Metabolic status was strongly related to BMI, with $28 \%$ of women with normal BMI being dysmetabolic compared with 55\% among overweight women and $76 \%$ among obese women $(P<0.0001)$.

\section{Angiographic CAD by Levels of BMI and Metabolic Status}

A consistent pattern was seen between BMI and metabolic status in relation to the prevalence of significant angiographic CAD (Table 2). Among women with normal metabolic status, the prevalence of significant angiographic CAD was $29 \%$ in those with normal BMI, $25 \%$ in overweight women, and $17 \%$ in obese women. These rates were much lower than those 
TABLE 2. Relationship Between BMI, Metabolic Status, and Prevalence of Significant Angiographic CAD

\begin{tabular}{lccccccc}
\hline & Metabolic & & Prevalence & Unadjusted & Adjusted & & \\
BMI Status* & Status & $\mathrm{n}$ & of CAD, $\%$ & OR & OR $\neq$ & $95 \% \mathrm{Cl}$ & $P$ \\
\hline Normal & Normal & 131 & 29.0 & 1.0 & 1.0 & $\ldots$ & $\ldots$ \\
Normal & Dysmetabolic & 50 & 56.0 & 3.12 & 3.11 & $1.50-6.41$ & 0.002 \\
Overweight & Normal & 120 & 25.0 & 0.82 & 1.04 & $0.58-1.89$ & 0.87 \\
Overweight & Dysmetabolic & 148 & 52.0 & 2.65 & 2.63 & $1.54-4.50$ & 0.0004 \\
Obese & Normal & 75 & 17.3 & 0.51 & 0.66 & $0.31-1.39$ & 0.27 \\
Obese & Dysmetabolic & 247 & 42.1 & 1.78 & 1.91 & $1.17-3.14$ & 0.01 \\
\hline
\end{tabular}

${ }^{*}$ Normal BMI status indicates BMI $\leq 24.9$; Overweight, BMl 25 to $\leq 29.9$; Obese, BMI $\geq 30$.

†Dysmetabolic indicates metabolic syndrome or diabetes.

$\ddagger$ Adjusted for age, race, menopausal status, and physical activity; 15 cases were excluded because of missing covariate data.

documented in dysmetabolic women $(56 \%, 52 \%$, and $42 \%$, respectively). These relations of metabolic status, but not BMI, being associated with prevalence of significant angiographic CAD persisted and remained statistically significant after adjustment for age, race, menopausal status, and physical activity. Specifically, compared with normal-weight women with normal metabolic status, dysmetabolic women who were normal weight, overweight, or obese had approximately 3.1, 2.6, and 1.9 times higher adjusted odds of having significant angiographic CAD.

\section{Cardiovascular Events by Levels of BMI and Metabolic Status}

A similar pattern of risk was observed when BMI was stratified by metabolic status and its association with cardiovascular events. Among women with normal metabolic status, 3-year survival rates were $95.8 \%$ in those with normal BMI, $97.8 \%$ in those who were overweight, and $98.7 \%$ in those who were obese (Figure 1). These survival rates were markedly higher than those observed in dysmetabolic women (normal BMI 86.6\%, overweight $91.9 \%$, obese $92.3 \%$ ). Thus, the 3-year survival rate was significantly higher in women with normal metabolic status than in those who were dysmetabolic $(97.2 \%$ versus $91.5 \%, P=0.003)$, with consistently higher survival observed at all levels of BMI. Adjusted 3-year risks of death were also in the direction of higher risk being associated with a dysmetabolic status $(\approx 2$-fold) but not BMI, yet this did not attain statistical significance, perhaps owing to low statistical power when the study cohort was stratified by levels of both BMI and metabolic status (Table 3). Similar results were observed for 3-year event-free rates from MACE (Figure 2; Table 3). Thus, dysmetabolic status, but not increasing levels of BMI, was associated with an increased risk of cardiovascular events. Moreover, within levels of metabolic status, obese and overweight individuals appeared to have nominally better 3-year event-free survival than those with normal BMI.

\section{Inflammation by Levels of BMI and Metabolic Status}

As a possible mechanism linking disturbed metabolic status, independent of body weight, with cardiovascular events, levels of inflammation (hs-CRP) were explored. At all levels of BMI, the prevalence of women with baseline hs-CRP values $\geq 1.5 \mathrm{mg} / \mathrm{dL}$ was highest among those who were dysmetabolic compared with those with normal metabolic status (Figure 3). This difference in inflammatory activity was particularly striking in overweight women, with only $4.9 \%$ of those with normal metabolic status having hs-CRP $\geq 1.5 \mathrm{mg} / \mathrm{dL}$ compared with $21.8 \%$ of women with disturbed

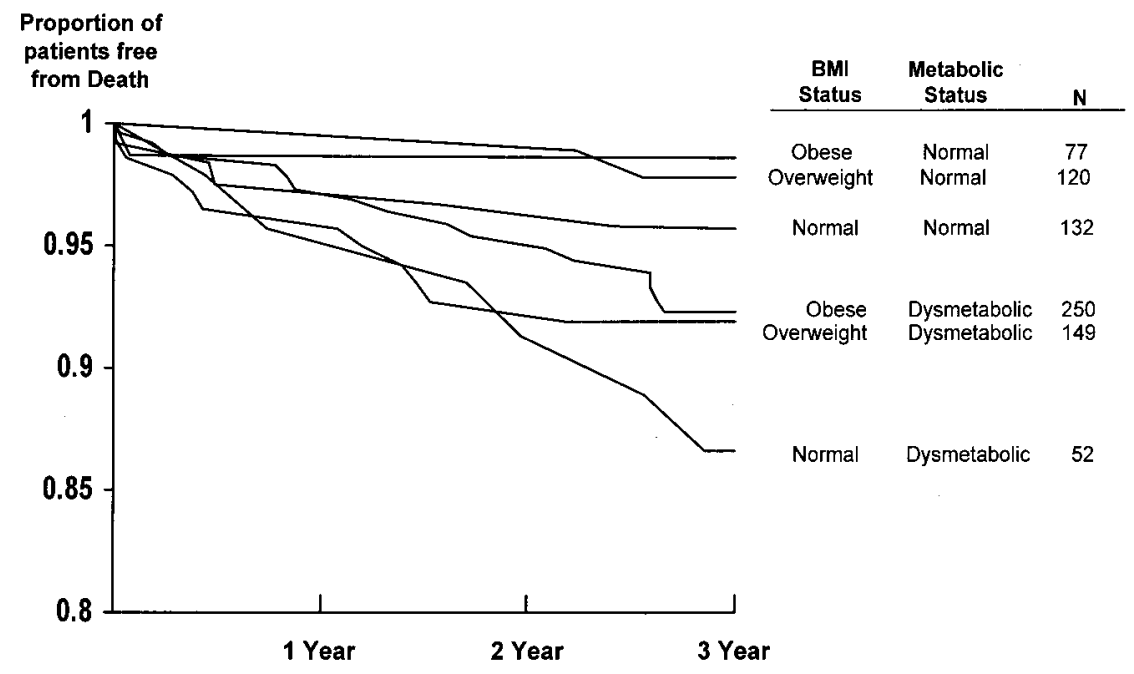

Figure 1. Kaplan-Meier curves of 3-year freedom from death by BMI and metabolic status at study entry. Dysmetabolic indicates metabolic syndrome or diabetes. 
TABLE 3. Relationship Between BMI, Metabolic Status, and 3-Year Risk of Death and MACE

\begin{tabular}{|c|c|c|c|c|c|c|c|}
\hline BMI Status* & Metabolic Status $\dagger$ & $\mathrm{n}$ & $\begin{array}{c}\text { 3-Year } \\
\text { Event Rate, } \\
\% \\
\end{array}$ & $\begin{array}{c}\text { Unadjusted } \\
\text { HR } \\
\end{array}$ & $\begin{array}{c}\text { Adjusted } \\
\text { HR } \ddagger\end{array}$ & $\begin{array}{c}95 \% \\
\mathrm{Cl}\end{array}$ & $P$ \\
\hline \multicolumn{8}{|c|}{ 3-year risk of death } \\
\hline Normal & Normal & 132 & 4.2 & 1.0 & 1.0 & $\cdots$ & $\cdots$ \\
\hline Normal & Dysmetabolic & 52 & 13.4 & 3.10 & 2.32 & $0.60-8.92$ & 0.22 \\
\hline Overweight & Normal & 120 & 2.2 & 0.45 & 0.83 & $0.15-4.63$ & 0.83 \\
\hline Overweight & Dysmetabolic & 149 & 8.1 & 1.98 & 1.79 & $0.55-5.80$ & 0.33 \\
\hline Obese & Normal & 77 & 1.3 & 0.36 & 0.66 & $0.07-6.01$ & 0.71 \\
\hline Obese & Dysmetabolic & 250 & 7.7 & 1.77 & 2.08 & $0.68-6.40$ & 0.20 \\
\hline \multicolumn{8}{|c|}{ 3-year risk of MACE } \\
\hline Normal & Normal & 132 & 9.6 & 1.0 & 1.0 & $\cdots$ & $\cdots$ \\
\hline Normal & Dysmetabolic & 52 & 26.0 & 2.99 & 2.21 & $0.81-5.59$ & 0.13 \\
\hline Overweight & Normal & 120 & 4.1 & 0.40 & 0.76 & $0.23-2.56$ & 0.66 \\
\hline Overweight & Dysmetabolic & 149 & 18.4 & 2.09 & 1.88 & $0.81-4.23$ & 0.14 \\
\hline Obese & Normal & 77 & 4.6 & 0.48 & 0.74 & $0.19-2.84$ & 0.66 \\
\hline Obese & Dysmetabolic & 250 & 17.7 & 1.97 & 2.04 & $0.93-4.48$ & 0.08 \\
\hline
\end{tabular}

HR indicates hazard ratio.

*Normal BMl status indicates BMl $\leq 24.9$; overweight, BMI 25 to $\leq 29.9$; and obese, BMl $\geq 30$.

†Dysmetabolic indicates metabolic syndrome or diabetes.

†For 3-year risk of death, HR was adjusted for age, race, prior myocardial infarction, chronic obstructive pulmonary disease, and number of lesions with $\geq 50 \%$ stenosis; 33 cases were excluded because of missing covariate data. For 3-year risk of MACE, HR was adjusted for age, race, prior myocardial infarction, chronic obstructive pulmonary disease, history of congestive heart failure, number of lesions with $\geq 50 \%$ stenosis, and physical activity level; 47 cases were excluded because of missing covariate data.

metabolic status $(P=0.0003)$. The cutpoint of hs-CRP $\geq 1.5$ $\mathrm{mg} / \mathrm{dL}$ corresponded to the upper $15 \%$ of the hs-CRP distribution.

\section{Independent Effect of BMI Versus Metabolic Status on Cardiovascular Events}

In both unadjusted and adjusted analyses (Table 4), unit increases in BMI (from normal to overweight to obese) were not associated with 3-year risk of death (adjusted hazard ratio $0.92,95 \%$ CI 0.59 to $1.51, P=0.69$ ) or MACE (adjusted hazard ratio $0.95,95 \% \mathrm{CI} 0.71$ to $1.27, P=0.73)$. In contrast, each unit increase in metabolic status (from normal to metabolic syndrome to diabetes) was associated with $\approx 2$-fold adjusted risk of death (hazard ratio 2.01, 95\% CI 1.26 to 3.20, $P=0.003$ ) and MACE (hazard ratio $1.88,95 \%$ CI 1.38 to $2.57, P<0.0001)$. Thus, the adverse effect of being dysmetabolic on long-term risk of cardiovascular events was independent of BMI, whereas being overweight or obese did not independently confer increased cardiovascular risk. These results were essentially unchanged when adjusting for baseline levels of hs-CRP (data not shown), thereby indicating that the adverse effect associated with dysmetabolic status was not principally due to (ie, confounded by) higher levels of inflammation in women who were dysmetabolic. Higher hs-CRP levels (log transformed) were suggestive of but not independently associated with 3-year risk of death (adjusted

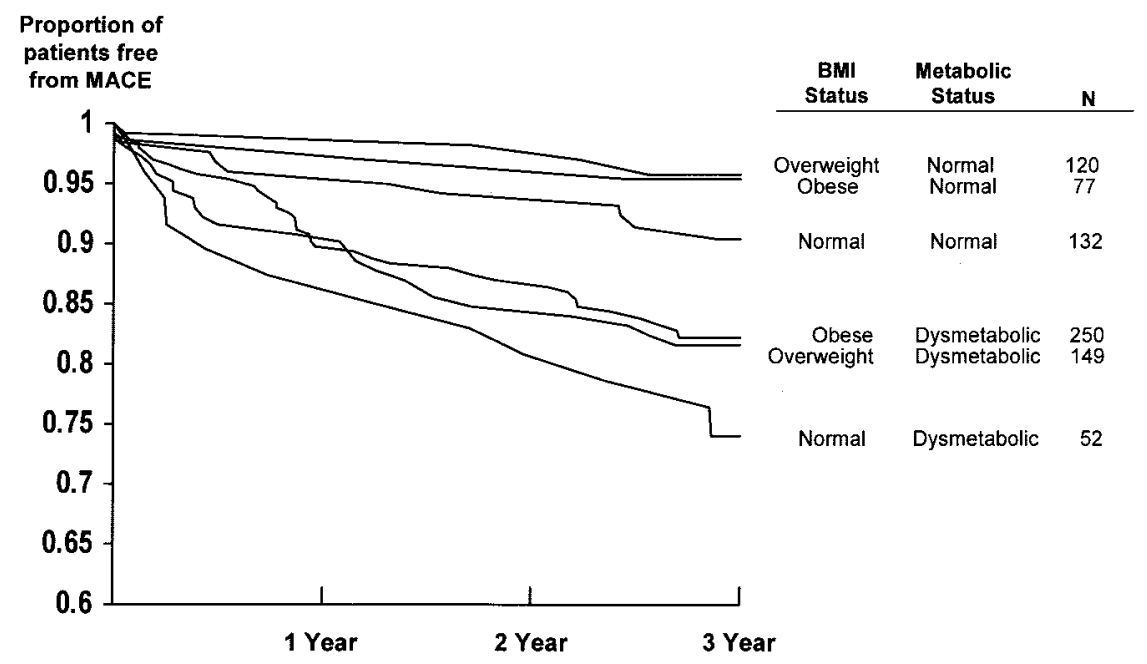

Figure 2. Kaplan-Meier curves of 3-year freedom from MACE (death, myocardial infarction, stroke, or congestive heart failure) by BMI and metabolic status at study entry. Dysmetabolic indicates metabolic syndrome or diabetes. 


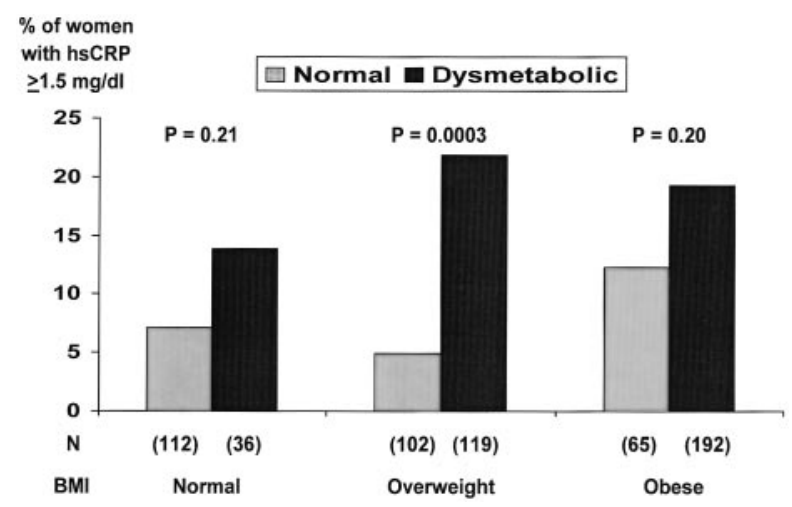

Figure 3. Percentage of women with baseline hs-CRP levels $\geq 1.5 \mathrm{mg} / \mathrm{dL}$ by BMI and metabolic status at study entry. Cutpoint of hs-CRP $\geq 1.5 \mathrm{mg} / \mathrm{dL}$ corresponds to upper $15 \%$ of hs-CRP distribution. Dysmetabolic indicates metabolic syndrome or diabetes.

hazard ratio $1.25,95 \% \mathrm{CI} 0.92$ to $1.68, P=0.15)$ or MACE (adjusted hazard ratio $1.21,95 \%$ CI 0.97 to $1.50, P=0.09$ ).

\section{Discussion}

The prevalence of obesity, insulin resistance, and type 2 diabetes has increased steadily in the United States over the past 2 decades to the point of being viewed as epidemic. ${ }^{1}$ In the present study of 780 women with suspected myocardial ischemia who were referred for coronary angiography, we observed a predictable but partially discordant relationship between increasing levels of BMI (normal versus overweight versus obese) and disturbed metabolic status (normal versus metabolic syndrome versus diabetes). Specifically, a minority (24\%) of obese women did not have the metabolic syndrome or diabetes, whereas a parallel minority of women with normal BMI (28\%) were classified as dysmetabolic (ie, with metabolic syndrome or diabetes). Abnormal metabolism was independently associated with a significantly increased risk of death or MACE. In contrast, increased BMI (ie, overweight and obesity) was not an independent predictor of cardiovascular risk.

Previous epidemiological studies have reported that obesity is an independent cardiovascular risk factor. ${ }^{7-10}$ However, the pathophysiological mechanism of this association is uncertain. The present data suggest that abnormal metabolism, manifested by the metabolic syndrome or diabetes, may explain in part this observation. This possibility is biologically plausible because central adiposity, which is a common manifestation of obesity, is associated with insulin resistance, which appears to be the underlying cause of the metabolic syndrome and type II diabetes. Furthermore, adipocytes produce proinflammatory cytokines, which might explain our and other observations of associations between abnormal metabolism and nonspecific inflammatory markers such as hs-CRP. ${ }^{22}$

TABLE 4. Relationship Between Baseline BMI,* Metabolic Status, $\dagger$ and Other Variables on 3-Year Risk of Death and MACE

\begin{tabular}{lccccc}
\hline & $\begin{array}{c}\text { Unadjusted HR } \\
(\mathrm{n}=780)\end{array}$ & $\begin{array}{c}\text { Adjusted HR } \\
(\mathrm{n}=780)\end{array}$ & $\begin{array}{c}\text { Adjusted HR } \\
(\mathrm{n}=747) \ddagger\end{array}$ & $95 \% \mathrm{Cl}$ & $P$ \\
\hline 3-year risk of death & & & & & \\
BMI* & 0.72 & 0.74 & 0.92 & $0.59-1.41$ & 0.69 \\
Metabolic status $\dagger$ & 2.47 & 2.39 & 2.01 & $1.26-3.20$ & 0.003 \\
Age (per 10 years) & $\ldots$ & 1.17 & 1.07 & $0.80-1.42$ & 0.66 \\
White & $\ldots$ & 0.88 & 0.75 & $0.55-5.80$ & 0.44 \\
History of COPD & $\ldots$ & $\ldots$ & 5.03 & $2.28-11.08$ & $<0.0001$ \\
Prior MI & $\ldots$ & $\ldots$ & 1.91 & $0.97-3.76$ & 0.06 \\
No. of lesions $>50 \%$ stenosis & $\ldots$ & $\ldots$ & 1.19 & $1.00-1.40$ & 0.04 \\
3-year risk of MACE & & & & & \\
BMI* & 0.79 & 0.79 & 0.95 & $0.71-1.27$ & 0.73 \\
Metabolic status $\dagger$ & 2.38 & 2.28 & 1.88 & $1.38-2.57$ & $<0.0001$ \\
Age (per 10 years) & $\ldots$ & 1.11 & 0.97 & $0.80-1.18$ & 0.77 \\
White & $\ldots$ & 0.77 & 0.83 & $0.51-1.36$ & 0.46 \\
History of COPD & $\ldots$ & $\ldots$ & 3.83 & $2.14-6.84$ & $<0.0001$ \\
History of CHF & $\ldots$ & $\ldots$ & 2.92 & $1.73-4.94$ & $<0.0001$ \\
Prior MI & $\ldots$ & $\ldots$ & 1.07 & $0.66-1.74$ & 0.78 \\
No. of lesions $>50 \%$ stenosis & $\ldots$ & $\ldots$ & 1.22 & $1.09-1.37$ & 0.0007 \\
Physical activity level§ & $\ldots$ & $\ldots$ & 0.67 & $0.48-0.94$ & 0.02 \\
\hline
\end{tabular}

COPD indicates chronic obstructive pulmonary disease; MI, myocardial infarction; CHF, congestive heart failure; and $H R$, hazard ratio.

${ }^{*}$ Coded as a 3-level variable: normal $=\mathrm{BMI} \leq 24.9$ (value of 1); overweight $=\mathrm{BMI} 25$ to $\leq 29.9$ (value of 2); and obese $=\mathrm{BMI} \geq 30$ (value of 3 ).

†Coded as a 3-level variable: normal (value of 1); metabolic syndrome (value of 2); and diabetic (value of 3 ).

$\ddagger \mathrm{n}=733$ for 3 -year risk of MACE.

$\S$ Average of physical activity at work, home, and during leisure time in past 12 months ( 1 =inactive, $4=$ heavy). 
The present findings also indicate that normal-weight women with the metabolic syndrome have a significantly increased cardiovascular risk. Similarly, overweight and obese women with normal metabolism have a relatively low cardiovascular risk. These results suggest that the clinical evaluation of abnormal metabolism (ie, metabolic syndrome and diabetes) should play a more important role than the diagnosis of obesity per se in cardiovascular risk stratification in women. One possible explanation for this finding is that measurement of BMI to define overweight and obesity does not quantify the magnitude or ratio of subcutaneous to visceral fat in a given individual. The visceral fat area, which is associated with an insulin-resistant state, ${ }^{27}$ appears to be an important link between many components of the metabolic syndrome, such as dyslipidemia and hypertension. ${ }^{28}$ Furthermore, classification of weight status by measurements of waist and hip circumference, the ratio of which may provide a clinically useful estimation of the proportion of abdominal or upper-body fat, ${ }^{29}$ also does not distinguish between accumulations of visceral and subcutaneous abdominal fat. Therefore, classification of weight status by routine measurements may underestimate the role of visceral fat distribution in the prediction of cardiovascular risk. This limitation of standard clinical measurements is germane to the present study because we do not know whether the present cohort of women with normal BMI and the metabolic syndrome had relatively high levels of visceral fat or, conversely, whether obese women with normal metabolic status had relatively low levels of visceral fat. Nevertheless, the present data suggest that classification of metabolic status by a simple and wellestablished clinical algorithm is more accurate than assessment of weight status alone for the stratification of cardiovascular risk in women.

The present study also demonstrated that inflammatory activity, as measured by levels of hs-CRP, was more closely associated with metabolic status than body weight. This finding is not fully intuitive, because adipose tissue is a source for the production and release of cytokines such as tumor necrosis factor- $\alpha$, IL- $1 \beta$, and IL- $6,{ }^{30}$ which induce hepatic production of hs-CRP, ${ }^{31}$ and because BMI has recently been shown to be a particularly strong correlate of hs-CRP. ${ }^{32}$ However, cytokines may also directly impede insulin-stimulated glucose uptake, ${ }^{33}$ which is consistent with the proposition that chronic subclinical inflammation should be considered a component of the definition of the metabolic syndrome. ${ }^{19}$ Moreover, when evaluated above and beyond the effects of BMI and the metabolic syndrome, we found a trend toward an association between levels of hs-CRP and risk of cardiovascular events.

\section{Study Limitations}

Our study population of women with suspected myocardial ischemia who were referred for coronary angiography is highly selective; the present results may not be generalizable to women at large or to men. Furthermore, selection bias may have been present. Specifically, we found that (1) among women with normal metabolic status, only $17 \%$ of those classified as obese had significant angiographic CAD compared with $29 \%$ of those with normal BMI, and (2) the prevalence of smoking was lowest in obese women. Thus, we cannot rule out a possible differential survival effect whereby smoking and/or the presence of angiographic CAD is more lethal in obese women. Similarly, it is possible that the perception of chest pain differs by body weight such that overweight and obese women seek diagnosis and treatment at lower (earlier) levels of ischemia and obstructive CAD than women with normal BMI. Finally, statistical power was limited when simultaneously stratifying the study cohort by levels of BMI and metabolic status; this precluded our ability to demonstrate statistical significance for apparent clinically relevant risks (ie, 2-fold) of cardiovascular events.

\section{Conclusions}

In women with suspected myocardial ischemia, the presence of the metabolic syndrome is highly prognostic of future cardiovascular risk, whereas measurement of BMI alone appears to confer little independent value in cardiovascular risk stratification. Therefore, the evaluation of metabolic status with the NCEP ATP III guidelines ${ }^{11}$ should be considered in all women, regardless of weight status. Although it remains prudent to recommend weight loss in overweight and obese women, control of all modifiable risk factors in both normal and overweight persons to prevent the transition to or reduce the manifestations of the metabolic syndrome should be considered the ultimate goal. Future studies of cardiovascular risk should not only incorporate the classification of metabolic status but should also evaluate the role of inflammatory activity as a potential mediator of the epidemiological association among obesity, metabolic status, and cardiovascular risk.

\section{Acknowledgments}

This study was supported by contracts from the National Heart, Lung, and Blood Institute (N01-HV-68161, N01-HV-68162, N01HV-68163, and N01-HV-68164).

\section{References}

1. Flegal KM, Carroll MD, Ogden CL, et al. Prevalence and trends in obesity among US adults, 1999-2000. JAMA. 2002;288:1723-1727.

2. National Center for Health Statistics. Healthy weight, overweight, and obesity among persons 20 years of age and over, according to sex, age, race, and Hispanic origin: United States, 1960-62, 1971-74, 1976-80, 1988-94, and 1999-2000. Available at: http://www.cdc.gov/nchs/data/ hus/tables/2002/02hus070.pdf. Accessed October 2003.

3. Higgins M, Kannel W, Garrison R, et al. Hazards of obesity: the Framingham experience. Acta Med Scand Suppl. 1988;723:23-36.

4. Han TS, van Leer EM, Seidell JC, et al. Waist circumference action levels in the identification of cardiovascular risk factors: prevalence study in a random sample. BMJ. 1995;311:1401-1405.

5. Pradhan AD, Skerrett PJ, Manson JE. Obesity, diabetes, and coronary risk in women. J Cardiovasc Risk. 2002;9:323-330.

6. Al Suwaidi J, Higano ST, Holmes DR Jr, et al. Obesity is independently associated with coronary endothelial dysfunction in patients with normal or mildly diseased coronary arteries. J Am Coll Cardiol. 2001;37: 1523-1528.

7. Manson JE, Willett WC, Stampfer MJ, et al. Body weight and mortality among women. $N$ Engl J Med. 1995;333:677-685.

8. Rissanen A, Knekt P, Heliovaara M, et al. Weight and mortality in Finnish women. J Clin Epidemiol. 1991;44:787-795.

9. Prineas RJ, Folsom AR, Kaye SA. Central adiposity and increased risk of coronary artery disease mortality in older women. Ann Epidemiol. 1993; $3: 35-41$.

10. Bengtsson C, Bjorkelund C, Lapidus L, et al. Associations of serum lipid concentrations and obesity with mortality in women: 20 year follow up of 
participants in prospective population study in Gothenburg, Sweden. BMJ. 1993;307:1385-1388.

11. Expert Panel on Detection, Evaluation, and Treatment of High Blood Cholesterol in Adults. Executive Summary of the Third Report of the National Cholesterol Education Program (NCEP) Expert Panel on Detection, Evaluation, and Treatment of High Blood Cholesterol in Adults (Adult Treatment Panel III). JAMA. 2001;285:2486-2497.

12. Ford ES, Giles WH, Dietz WH. Prevalence of the metabolic syndrome among US adults: findings from the third National Health and Nutrition Examination Survey. JAMA. 2002;287:356-359.

13. Laaksonen DE, Lakka HM, Niskanen LK, et al. Metabolic syndrome and development of diabetes mellitus: application and validation of recently suggested definitions of the metabolic syndrome in a prospective cohort study. Am J Epidemiol. 2002;156:1070-1077.

14. Alexander CM, Landsman PB, Teutsch SM, et al, for the Third National Health and Nutrition Examination Survey (NHANES III), National Cholesterol Education Program (NCEP). NCEP-defined metabolic syndrome, diabetes, and prevalence of coronary heart disease among NHANES III participants age 50 years and older. Diabetes 2003;52:1210-1214.

15. Wilson PW, Kannel WB, Silbershatz H, et al. Clustering of metabolic factors and coronary heart disease. Arch Intern Med. 1999;159: 1104-1109.

16. Reaven GM. Banting lecture 1988: role of insulin resistance in human disease. Diabetes. 1988;37:1595-1607.

17. Kaplan NM. The deadly quartet: upper-body obesity, glucose intolerance, hypertriglyceridemia, and hypertension. Arch Intern Med. 1989;149: $1514-1520$.

18. Hanley AJ, Karter AJ, Festa A, et al. Factor analysis of metabolic syndrome using directly measured insulin sensitivity: the Insulin Resistance Atherosclerosis Study. Diabetes. 2002;51:2642-2647.

19. Rader DJ. Inflammatory markers of coronary risk. N Engl J Med. 2000; 343:1179-1182.

20. Sattar N, Gaw A, Scherbakova O, et al. Metabolic syndrome with and without C-reactive protein as a predictor of coronary heart disease and diabetes in the West of Scotland Coronary Prevention Study. Circulation. 2003;108:414-419.

21. Pearson TA, Mensah GA, Alexander RW, et al. Markers of inflammation and cardiovascular disease: application to clinical and public health practice: a statement for healthcare professionals from the Centers for
Disease Control and Prevention and the American Heart Association. Circulation. 2003;107:499-511.

22. Han TS, Sattar N, Williams K, et al. Prospective study of C-reactive protein in relation to the development of diabetes and metabolic syndrome in the Mexico City Diabetes Study. Diabetes Care. 2002;25: 2016-2021.

23. Bairey Merz CN, Kelsey SF, Pepine CJ, et al. The Women's Ischemia Syndrome Evaluation (WISE) study: protocol design, methodology and feasibility report. J Am Coll Cardiol. 1999;33:1453-1461.

24. NHLBI Obesity Education Initiative Expert Panel on the Identification, Evaluation, and Treatment of Overweight and Obesity in Adults. Clinical Guidelines on the Identification, Evaluation, and Treatment of Overweight and Obesity in Adults. Bethesda, Md: National Heart, Lung, and Blood Institute; 1998.

25. Ridker PM, Rifai N, Rose L, et al. Comparison of C-reactive protein and low-density lipoprotein cholesterol levels in the prediction of first cardiovascular events. $N$ Engl J Med. 2002;347:1557-1565.

26. Sharaf B, Pepine CJ, Kerensky RA, et al. Detailed angiographic analysis of women with suspected ischemic chest pain (pilot phase data from the NHLBI-sponsored Women's Ischemia Syndrome Evaluation [WISE] Study Angiographic Core Laboratory). Am J Cardiol. 2001;87:937-941.

27. Pouliot MC, Despres JP, Nadeau A, et al. Visceral obesity in men: associations with glucose intolerance, plasma insulin and lipoprotein levels. Diabetes. 1992;41:826-834.

28. Nagaretani H, Nakamura T, Funahashi T, et al. Visceral fat is a major contributor for multiple risk factor clustering in Japanese men with impaired glucose tolerance. Diabetes Care. 2001;24:2127-2133.

29. Bjorntorp P. Obesity and risk of cardiovascular disease. Ann Clin Res. 1985;17:3-9.

30. Mohamed-Ali V, Pinkney JH, Coppack SW. Adipose tissue as an endocrine and paracrine organ. Int J Obes Relat Metab Disord. 1998;22: $1145-1158$.

31. Papanicolaou DA, Wilder RL, Manolagas SC, et al. The pathophysiologic roles of interleukin-6 in human disease. Ann Intern Med. 1998;128: 127-137.

32. Rexrode KM, Pradhan A, Manson JE, et al. Relationship of total and abdominal adiposity with CRP and IL-6 in women. Ann Epidemiol. 2003;13:674-682.

33. Youd JM, Rattigan S, Clark MG. Acute impairment of insulin-mediated capillary recruitment and glucose uptake in rat skeletal muscle in vivo by TNF- $\alpha$. Diabetes. 2000;49:1904-1909. 